\title{
Circumstellar disk evolution: Constraining theories of planet formation
}

\author{
Michael R. Meyer \\ Steward Observatory, The University of Arizona \\ 933 N. Cherry Avenue, Tucson, AZ 85721 (USA)
}

\begin{abstract}
Observations of circumstellar disks around stars as a function of stellar properties such as mass, metallicity, multiplicity, and age, provide constraints on theories concerning the formation and evolution of planetary systems. Utilizing ground- and space-based data from the far-UV to the millimeter, astronomers can assess the amount, composition, and location of circumstellar gas and dust as a function of time. We review primarily results from the Spitzer Space Telescope, with reference to other ground- and space-based observations. Comparing these results with those from exoplanet search techniques, theoretical models, as well as the inferred history of our solar system, helps us to assess whether planetary systems like our own, and the potential for life that they represent, are common or rare in the Milky Way galaxy.
\end{abstract}

Keywords. solar system: formation, stars: circumstellar matter, pre-main-sequence, planetary systems: protoplanetary disks, planetary systems: formation

\section{Introduction}

Are there multitudes of planetary systems capable of harboring life, like our own Solar System? Answering this question motivates the research activities of a great number of astronomers, as well as scientists of many disciplines. Yet the answer depends on which aspect of our solar system to which one is comparing the physical properties of other systems. Extrapolation of radial velocity results to $20 \mathrm{AU}$ suggests that planets with mass at least a third that of Jupiter's surround 15-20\% of sun-like stars (Cumming et al. 2008). Yet lower mass planets might turn out to be even more common (e.g. Mayor et al. 2009). Enormous progress has been made in the past several years on many aspects of circumstellar disk evolution (e.g. Meyer et al. 2007), especially those that can be addressed with observations from the Spitzer Space Telescope (Werner et al. 2006). In this review we explore answers to three key questions: 1) What is the time available to form gas giant planets? 2) What is the history of planetesimal collisions versus radius? 3) How do answers to the above vary with stellar properties? Because the answers to these questions are subtle, one needs large stellar samples with reliable stellar ages from the youngest pre-main sequence stars to the oldest stars known in the galactic disk. In our attempt to study important evolutionary processes in the formation and evolution of planetary systems, we assemble groups of stars with like properties (such as a narrow range in stellar mass) as a function of age, the main topic of this symposium. We hope that by studying the mean (as well as the dispersion) in those properties of the circumstellar environment as a function of time, we can create a "movie" (or range of plausible trajectories) that helps tell the story of how our solar system might have formed. Once completed for one range in stellar mass, we can attempt to repeat the study for other stellar masses. Examining the differences in circumstellar disk evolution as a function of stellar mass may be our best tool in delineating the most important physical processes in planet formation. It is a lofty goal, and often strong assumptions 
are required to make progress. We can only hope that most of these assumptions represent hypotheses we can test in the near future.

Observations of circumstellar gas and dust, both its amount and geometrical distribution, can be compared to theoretical timescales for its expected evolution. Keplerian orbits can range from days to millennia. The viscous timescale in the context of an $\alpha$ disk model depends on the orbital radius and can be $<1$ Myr within 10 AU for reasonable parameters (Hartmann 1998). Preliminary results suggest that disk chemistry proceeds more slowly than relevant dynamical times indicating that mixing could be important (Bergin et al. 2007). The inward migration of solids in the disk results in the loss of planet-building material and remains a serious problem on many scales: a) gas drag on meter-sized bodies can reach 1 AU/century (Weidenschilling, 1977); b) Type I migration of lunar-mass planetary embryos on timescales of $10^{5}$ yrs; and c) Type II migration of forming gas giant planets on timescales proportional to the viscous time (e.g. Ida \& Lin, 2008 and references therein). The timescale for orderly growth of bodies through collisions (e.g. Goldreich et al. 2004) is proportional to the product of the radius and volume density of typical particles divided by the product of the mass surface density of solids and the orbital frequency. The timescale for radiogenic heating of forming planetesimals is set by the relative abundances of radioactive nuclides as well their half-lives (Sanders \& Taylor, 2005). Current models suggest that photoevaporation of gas from illumination by EUV, FUV, and $\mathrm{x}$-ray emission can disperse primordial gas disks around sun-like stars on timescales < 10 Myr (Gorti \& Hollenbach 2009; Ercolano et al. 2009). Additional physical processes are important in debris disk evolution. In most (if not all) debris disks studied to date (our solar system dust disk being a notable exception), mutual collisions between dust grains reduce particle sizes to the blow-out limit where radiation pressure efficiently removes them from the system (Wyatt 2008). The Lyapunov timescale characterizes divergence of orbital elements in a chaotic system and is related to the timescale for instability in planetary systems (Murison et al. 1994). In this context, we note that our own planetary system is thought to be stable on timescales comparable to its present age of 4.56 Gyr (Laskar 1994) while the main sequence lifetime of the Sun is approximately twice this.

In what follows, we describe some of the observational evidence for primordial disk evolution and resulting constraints on theories of planet formation. We follow with a brief discussion of planet formation, the evolution of planetesimals belts, and the dust debris they generate. We conclude with a summary and look forward to exciting developments we can anticipate in the years ahead.

\section{Primordial circumstellar disks}

It is now well established that most sun-like stars form surrounded by circumstellar disks. These disks are primordial mixtures of gas and dust initially resembling the composition of the interstellar medium from which the star-disk systems form. Near-infrared excess emission traces the hottest inner disk structures within $0.1 \mathrm{AU}$. While the inner edge of the dust disk is determined by the location at which the dust particles sublimate ( $1400 \mathrm{~K}$ for typical silicates; Muzerolle et al. 2003), the gas disk can extend inward, perhaps terminating near the boundary set by magnetospheric accretion theory (e.g. Shu et al. 1994). The pioneering work of Strom et al. (1989) suggested that inner disks dissipate on timescales of 10 Myr from observations of K-band excess toward T Tauri stars in the Taurus dark cloud. Haisch et al. (2001) surveyed near-IR excess emission using colorcolor diagrams toward hundreds of stars in several young clusters concluding that the mean inner disk lifetime is approximately $3 \mathrm{Myr}$. Yet if the spectral type of star is known, 
and multi-color optical/infrared photometry is available, one can carefully separate the effects of intrinsic stellar colors, reddening due to dust along the line of sight toward the star, and circumstellar disk excess determining with greater precision the magnitude of any excess emission (e.g. Meyer et al. 1997). This approach was used by Hillenbrand (2008) and colleagues to construct the evolutionary diagram shown in Figure 1 which we have adapted to include: a) relative formation timescales for solar system objects as determined from measurements of extinct radioactive nuclides from meteorite samples (Scott, 2007; Jacobsen, 2005); and b) the frequency distribution of inner disk lifetimes based on these evolutionary diagrams. While the mean disk lifetime is $3 \mathrm{Myr}$, there is a large dispersion of inner disk lifetimes. Andrews \& Williams (2005) have observed the distribution of disk masses for young stars ranges over two orders of magnitude from millimeter wave continuum data. Perhaps the distribution of initial conditions (specific angular momenta in collapsing cloud cores) results in a distribution of disk masses and thus disk lifetimes.

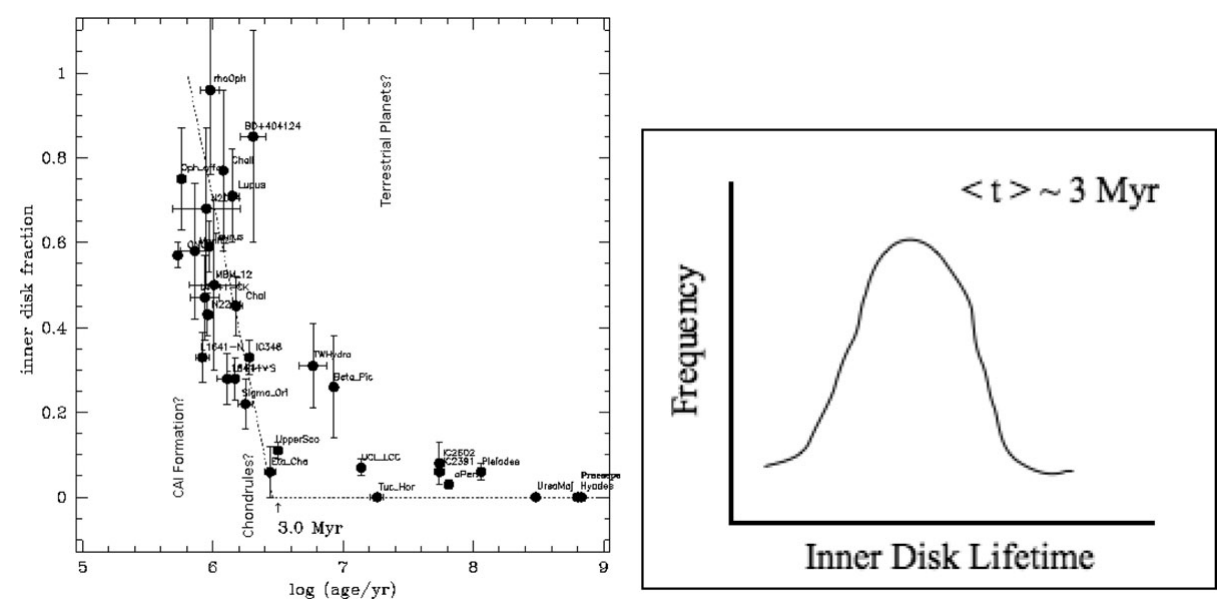

Figure 1. Left: Fraction of inner accretion disks as a function of time surrounding sun-like stars (roughly $0.5-2 \mathrm{M}_{\odot}$ ) as traced by near-IR excess emission observed toward stars with known spectral types. Figure adapted from Hillenbrand (2008) with the approximate timescales for the formation of solar system objects indicated from study of extinct radioactive nuclides. Right: Schematic representation of distribution of inner disk lifetimes obtained by subtracting adjacent bins in the left panel.

How long does it take for material in the inner disk to transition from optically-thick to optically-thin? Skrutskie et al. (1990) provided some preliminary answers based on $10 \mu \mathrm{m}$ observations of $\mathrm{T}$ Tauri stars. They found that most stars lacking $2 \mu \mathrm{m}$ excess also lacked mid-infrared excess emission. A small handful of objects, the so-called transition objects, exhibited modest mid-IR excess but lacked evidence for hot dust. From the ratio of the number of objects in transition compared to samples of T Tauri stars (few \% with respect to T Tauri stars with primordial accretion disks, CTTS, or a smaller fraction of young stars overall) times the typical age of the sample stars (1-3 Myr), they estimated the duration of the transition phase to be very short $\left(\sim 10^{5}\right.$ years $)$. Subsequent work has verified these estimates. For example, Silverstone et al. (2006) found no examples of inner dust disks in systems that lack signatures of on-going gas accretion from the disk onto the star (cf. Cieza et al. 2007). However with surveys of star-forming regions enabled by the Spitzer Space Telescope, dozens of these rare objects can now be identified (e.g. Merin et al. 2008). 
Much attention is focused on understanding the nature of transition disks (Najita et al. 2007; Alexander \& Armitage 2007). Some appear to have inner cleared regions due to the presence of previously unseen faint companions at small orbital radii (Ireland \& Kraus, 2008). Work continues to understand the detailed distribution of gas and dust in these systems (e.g. Espaillat et al. 2007). One troubling feature in all of these disk studies is the general lack of correlation between key physical variables and observational properties of the disks (Watson et al. 2009). For example, Pascucci et al. (2008) found no difference in dust properties between binary and single stars. Some disks can be quite long lived (more than $10 \mathrm{Myr}$ old) and still appear very similar to typically much younger classical T Tauri stars (e.g. PDS 66; Cortes et al. 2009). Often morphological ordering of disk spectra or other properties can be quite compelling (e.g. Bouwman et al. 2008). But more often than not, ranking by estimates of stellar age appear to disturb the apparent evolutionary sequences. Although errors in determining stellar ages are a likely culprit, it does seem that there are "hidden variables" contributing significantly to primordial disk evolution.

As a complement to studies of the dust, can we probe further the evolution of the gas from which giant planets might form? For young stars in the Taurus dark cloud, there is an excellent correspondence between spectroscopic signatures of gas accretion from the inner disk onto the surface of the star and the presence of near-IR excess emission (Hartigan, Edwards, and Ghandour, 1995). Indeed the evolution of accretion rates appears to mimic to some extent the evolution of hot dust (Hartmann et al. 1998; Gatti et al. 2008). Spitzer spectra are starting to reveal interesting chemistry in the disks young young stars (Carr \& Najita, 2008; Pascucci et al. 2009). High resolution nearinfrared spectroscopy combined with high spatial resolution can reveal offsets between the emitting regions of various molecules (Pontoppidan et al. 2008). Millimeter wave observations are required to trace rotational transitions of cool gas at large radii (e.g. Dutrey et al. 2007).

Yet the bulk of the mass in these disks is in molecular hydrogen, which is difficult to detect. Some efforts have been made to trace molecular hydrogen directly by observing the pure rotational lines in the mid-infrared from the ground at high spectral resolution (Bitner et al. 2008). The Spitzer Space Telescope also provided a platform to search for gas using the IRS in high resolution mode. Based on equilibrium chemical models of Gorti \& Hollenbach, 2004), Pascucci et al. (2006) report non-detections from the FEPS survey (Meyer et al. 2006) for stars with ages between 3-100 Myr that lack signatures of accretion, but possess optically-thin dust emission. The upper limits place constraints of $<10 \mathrm{Myr}$ on the timescale to form gas giants in these systems. These timescales are of interest for comparison to models of gas giant planet formation through classical core accretion (e.g. Lissauer \& Stevenson 2007) as well as gravitational fragmentation which proceeds more quickly (Durisen et al. 2007). Future observations with Herschel and SOFIA will be powerful probes of even small amounts of residual emission perhaps placing constraints on the formation of super-earths and ice giants. We note for completeness that circumstellar gas has been detected for some debris disks (e.g. Dent et al. 2005), which are the subject of the next section.

\section{Planet formation and generation of debris dust}

How does remnant disk material evolve when the bulk of the gas capable of forming giant planets has dissipated? In the classical theory, protoplanets initially grow in an orderly way through collisions of comparable sized bodies. As gravitational focusing becomes important, the larger bodies grow fastest in a runaway mode until a modest 
number of "successes" reach the local isolation mass, essentially consuming all material within several times their gravitational sphere of influence (Hill radius). These oligarchs then perturb each other over time resulting in handfuls of collisions that create a small number of planets (e.g. Nagasawa et al. 2007). At 1 AU around the Sun, we imagine the Earth as built through the collision of several Mars-sized objects. $\dagger$ It is thought that similar processes operate (in the presence of gas) to form the cores of giant planets. In the first phase, we can estimate the frequency of collisions by knowing the surface density of solids $(\sigma)$ as well as the orbital timescale $(\Omega)$ :

$$
\tau \sim\left(R_{b o d y} \times \rho_{b o d y}\right) /(\sigma \times \Omega)
$$

where $\mathrm{R}$ and $\rho$ are the radius and volume density of the object built through these collisions in time $\tau$. If we combine estimates of the dependence of disk mass on star mass $\left(\sigma_{\text {disk }} \sim M_{*}\right)$ and orbital radius $\left(\sigma_{\text {disk }} \sim 1 / a\right)$ with the orbital frequency we get $\tau \sim a^{5 / 2} M_{*}^{-3 / 2}$ for a constant $\rho$. This implies, within a fixed time interval, disks around stars of higher mass form: a) more massive planets at a given orbital distance; and b) planets of a given mass at larger orbital separation. Both are consistent with recent radial velocity results (Johnson et al. 2007). Yet it appears that primordial disks evolve more quickly around stars of higher mass (Carpenter et al. 2006), complicating the implications of this simple picture for planet formation around stars as a function of their mass.

Observations of debris disks have become powerful tools to study the evolution of planetary systems and their value has increased as we have learned more about the asteroid and Kuiper belts in our own Solar System (Wyatt 2008). As cooler dust orbiting at larger radii emits at longer wavelengths, we can use the wavelength dependence of excess emission as a proxy for its location in the absence of resolved images. With the Spitzer Space Telescope, we can detect $>10^{-5}$ Earth masses of dust if it is found in micron-sized particles from photometric surveys at $24 \mu \mathrm{m}$, efficiently tracing material with temperatures above $100 \mathrm{~K}$ (often tracing radii $<10 \mathrm{AU}$ around sun-like stars). Meyer et al. (2008) report the results from FEPS for $24 \mu \mathrm{m}$ excess emission around an unbiased parent sample of 309 stars. They find that 10-20\% of sun-like stars show evidence for $24 \mu \mathrm{m}$ excess over an age range from 3-300 Myr with a significant drop in the frequency of $24 \mu \mathrm{m}$ excess for older stars. As we can only observe the product of the frequency of this phenomena and its duration, the interpretation of these results is ambiguous. They also suggest that the frequency of excess may be higher for stars in open clusters compared to field stars of comparable age though the evidence for this is not significant. Note that the ages of the open cluster stars are the "gold standard" for stellar ages in these studies and thus it is difficult to compare results between them and field stars. In contrast, Greaves et al. (2009) find a surprising lack of emission at $1.3 \mathrm{~mm}$ towards sun-like stars in the Pleiades open cluster despite significant improvement in sensitivity compared to previous observations.

The above mentioned Spitzer results are consistent with other surveys for $24 \mu \mathrm{m}$ excess around FGK stars as a function of time (e.g. Siegler et al. 2007). It is worth remembering that these survey limits are only able to detect dust producing planetesimal belts that are about $\times 1000$ brighter than our own inner zodiacal dust generated from collisions in the asteroid belt. Wyatt et al. (2007) compare these observations to models of collisional evolution for planetesimal belts finding that some warm debris excesses (e.g. HD 69830) are so large that they cannot be the result of pure collisional evolution and must be transient. Although the models of Kenyon \& Bromley (2004) explain the observed behavior in a qualitative way, updates to the input physics continue to improve the agreement

$\dagger$ The last of these collisions resulting in the formation of the Moon (e.g. Canup 2004). 
between the models and data (Bromley \& Kenyon 2008). Models for several debris disks detected with Spitzer require extended distributions of dust (Hillenbrand et al. 2008) consistent with resolved images of some sources (Ardila et al. 2004).

What (if any) correlation should we expect between the presence of dust debris and the frequency of gas giant planets detected through radial velocity variations? One might speculate that a disk rich in heavy elements capable of forming gas giant planets (e.g. Fischer \& Valenti 2005; Santos et al. 2004) might initially have a high surface density of solids and thus produce a lot of dust early-on, outshining a system that lacked enough dust to form giant planets. If however, such a system produces several planets unstable to mutual perturbations, dynamical rearrangement could deplete dust-producing planetesimals in the inner and outer regions as suggested by the Nice model for the early evolution of our Solar system (e.g. Tsiganis et al. 2005). Such a system could then become a very weak dust producer at late times (Figure 2). It is in part these complexities that prevent us from making comparisons of Spitzer observations to what is known about our Solar system.

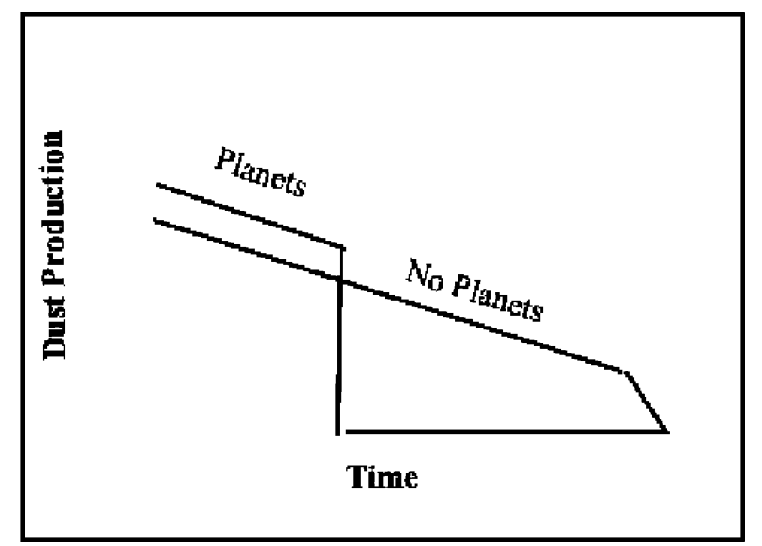

Figure 2. Graphical representation of dust production versus time for the case of a disk with an initially high surface density of solids that forms gas giant planets and another with a lower initial surface density that does not. The planet-forming disk outshines the other disk early on, but then suffers a dynamical event that removes most of the dust-generating planetesimals. The disk that was never able to form giant planets might generate more dust at late times.

Moro-Martin et al. (2007a) search for a correlation between the presence or absence of excess with gas giant planets and was unable to confirm any (though the sample size was modest). Apai et al. (2008) also searched (without success) for the presence of gas giant planets around stars for which large inner holes in the dust debris had been inferred. There are, however, remarkable examples of systems with evidence for planets that also maintain debris which deserve special study (Moro-Martin et al. 2007b; Lovis et al. 2006) including the recently announced direct imaging results for Fomalhaut (Kalas et al. 2008) and HR 8799 (Marois et al. 2008). We can also ask whether debris is correlated with the heavy element abundance of the star as it is famously with the probability of having a gas giant planet. Greaves, Fischer, \& Wyatt (2006) find that the metallicity distribution of stars with cold debris detected in the sub-millimeter is consistent with having been drawn from the same distribution as the parent sample of stars. We find a similar result for stars in the FEPS sample (Najita et al., in preparation). Perhaps gas giant planet formation is a threshold phenomena with regard to the heavy element content of the primordial circumstellar disk whereas the requirements for ending up with a dust producing debris 
disk are more forgiving. Bryden et al. (2006) estimate that Spitzer only detects the tip of the iceberg in terms of dust debris, and survey results to date are consistent with our Solar System IR emission being near the mean for sun-like stars in the Milky Way.

Given the mixed results for the correlation of debris with planets, can we expect more order in the behavior of debris with stellar multiplicity? There is considerable literature on the similarities and differences in disk evolution between single and binary $\mathrm{T}$ Tauri stars (Monin et al. 2007). With regard to debris around one or both components of a binary system, Trilling et al. (2007) report Spitzer results finding a complex mix of behaviors including circumbinary debris disks, circumstellar disks in wide binary systems, as well as the apparition of dust at inferred orbital distances consistent with the separation of the binary companion! At minimum, one can infer that debris dust as detected by Spitzer is not inhibited in multiple systems.

The "last word" from the FEPS program comes from Carpenter et al. $(2008 ; 2009)$ in which the extant database and synthesis of results are described respectively. With the addition of the IRS spectra from 5-33 $\mu \mathrm{m}$ for the sample, debris suspected from the $24 \mu \mathrm{m}$ photometry can be confirmed with confidence. Even more important, the temperature of the hottest dust detected can be estimated from the broadband spectrophotometry providing estimates of the inner radius of the debris disks in the sample. The detected dust is cooler on average (and thus located at larger radii) than assumed in Meyer et al. (2008): the distribution of inner radii for the (often extended) debris disks detected in the survey range from 3-30 AU, with a peak at $10 \mathrm{AU}$. There is no evidence for evolution of the dust temperature with age, though the magnitude of the observed excess does decline over time (with considerable dispersion at any one age). It appears that some of the $24 \mu \mathrm{m}$ excess emission observed is the Wien tail of bright, but cool, dust at radii beyond the terrestrial planet zone. Emission evolves from bright to faint (or in some cases hot to cool) within $300 \mathrm{Myr}$. Yet the picture that emerges overall is one where most debris is cleared out to radii of $10 \mathrm{AU}$ on timescales of 3-10 Myr! Whether this represents complete "mission success" for planet formation between $0.1-10$ AU or presents a challenge to current theory remains to be explored. Constraining the outer disk radii will require observations at longer wavelengths utilizing Herschel, SOFIA, or more sensitive sub-millimeter observations (e.g. Roccatagliati et al. 2009).

It is extremely tempting to compare these results for FKG stars to those for stars of different temperature \& luminosity in order to explore diversity in the formation and evolution of planetary systems as a function of stellar mass. However, such comparisons require caution. For example, in a survey where the sensitivity is limited by photometric precision of detecting an excess in contrast to the flux of the star, the amount of detectable dust around a more luminous star is larger than the detectable amount around one of lower luminosity. Also dust at a given temperature is located at a larger radius around a more luminous star. Any comparison should take this into account in terms of solid angle for the emission as well as the expected orbital period and dust surface density distribution. Overall, one can say that the magnitude of the excesses observed at 24 and $70 \mu \mathrm{m}$ around A stars are larger, and more common, than those observed around G stars ( $\mathrm{Su}$ et al. 2006), yet the timescale for the duration of the phenomena is shorter around A stars compared to G stars (Currie et al. 2007; cf. Trilling et al. 2008). Gautier et al. (2007) find that debris emission around M dwarfs is even weaker.

\section{Summary and future work}

So are planetary systems like our own are common or rare among sun-like stars in the Milky Way galaxy? Unfortunately, we cannot yet answer this question, but we can provide 
some important constraints that are stepping-stones to future progress. Primordial Disk Evolution: Disks around lower mass stars are less massive and live longer than their more massive counterparts. The large observed dispersion in evolutionary times could indicate a dispersion in initial conditions. Overall, disk evolution appears to proceed from inside-out as expected. Change you can believe in: The duration of the transition time from primordial to debris is $\sim 10^{5}$ yrs. Planetesimal belts evolve quickly out to $3-30$ AU. Evidence for $24 \mu \mathrm{m}$ excess is largely gone by $300 \mathrm{Myr}$. There is a hint that such excesses might be more common in open clusters at a given age though more work on this is needed. Debris Disk Evolution: Currently detectable extra-solar debris systems are all collision-dominated in their evolution. Debris is brighter and more common around stars of higher mass. Evolutionary paths are diverse but the observed distributions are consistent with our Solar System debris disk being common among stars $>1$ Gyr old. The connection between debris and planetary systems is unclear. Yet it may turn out that debris (and perhaps terrestrial planets) are more common than their gas giant counterparts. As we know that nearly all young stars begin their lives surrounded by primordial disks of gas and dust capable of making some sort of planetary system, one wonders: are systems without debris those with dynamically full planetary systems, or those without any planets whatsoever?

Anyone who has taken a turn leading the blind around the proverbial elephant (i.e. modelling spectral energy distributions) knows that a resolved image is worth more than $1024 \times 1024$ photometric points on an SED. Constraining model parameters with resolved emission at one or more wavelengths is vital to making progress. In addition to high contrast imaging of disks in scattered light from space and ground-based telescopes equipped with adaptive optics, millimeter wave interferometry will continue to make crucial contributions. The soon to be launched ESA Herschel Space Telescope will also build on the work of Spitzer, providing dozens of newly resolved debris disks in thermal emission. Finally, we look forward to science observations with ALMA and JWST that will expand our understanding of the formation and evolution of planetary systems in ways we can scarcely imagine (provided of course that we first solve the thorny problem of obtaining accurate stellar ages and uncover the nearest, youngest, sun-like stars as prime targets of observation).

\section{Acknowledgement}

The author would like to thank many colleagues whose work has contributed to his current understanding regarding the formation and evolution of planetary systems including members of the FEPS/c2D/Glimpse Legacy Science Teams, the MIPS/IRS/IRAC Instrument Teams, and the Spitzer Science Center staff. MRM acknowledges support from the Legacy Science Program through a contract from NASA/JPL as well as the LAPLACE node of the NASA Astrobiology Institute.

\section{References}

Alexander, R. D. \& Armitage, P. J. 2007, MNRAS, 375, 500

Andrews, S. M. \& Williams, J. P. 2005, ApJ, 631, 1134

Apai, D., et al. 2008, ApJ, 672, 1196

Ardila, D. R., et al. 2004, ApJ, 617, L147

Bergin, E., Aikawa, Y., Blake, G., \& van Dishoeck, E. 2007, Protostars \& Planets V, 751

Bitner, M. A., et al. 2008, ApJ, 688, 1326

Bouwman, J., et al. 2008, ApJ, 683, 479

Bryden, G., et al. 2006, ApJ, 636, 1098 
Canup, R. M. 2004, ARAA, 42, 441

Carpenter, J. M., Mamajek, E. E., Hillenbrand, L. A., \& Meyer, M. R. 2006, ApJ, 651, L49

Carpenter, J. M., et al. 2008, ApJS, 179, 423

Carpenter, J. M., et al. 2009, ApJS, 181, 197

Carr, J. S. \& Najita, J. R. 2008, Science, 319, 1504

Cieza, L., et al. 2007, ApJ, 667, 308

Cortes, S., et al. 2009, ApJ, in press (arXiv:0903.3801).

Cumming, A., Butler, R. P., Marcy, G. W., Vogt, S. S., Wright, J. T., \& Fischer, D. A. 2008, $P A S P, 120,531$

Currie, T., Kenyon, S. J., Balog, Z., Rieke, G., Bragg, A., \& Bromley, B. 2008, ApJ, 672, 558

Dent, W. R. F., Greaves, J. S., \& Coulson, I. M. 2005, MNRAS, 359, 663

Durisen, R. H., Boss, A. P., Mayer, L., Nelson, A. F., Quinn, T., \& Rice, W. K. M. 2007, Protostars and Planets V, 607

Dutrey, A., Guilloteau, S. \& Ho, P. 2007, Protostars and Planets V, 495

Ercolano, B., Drake, J. J., Raymond, J. C., \& Clarke, C. C. 2008, ApJ, 688, 398

Espaillat, C., Calvet, N., D’Alessio, P., Hernández, J., Qi, C., Hartmann, L., Furlan, E., \& Watson, D. M. 2007, ApJ, 670, L135

Fischer, D. A. \& Valenti, J. 2005, ApJ, 622, 1102

Gatti, T., Natta, A., Randich, S., Testi, L., \& Sacco, G. 2008, A\&A, 481, 423

Gautier, T. N., III, et al. 2007, ApJ, 667, 527

Goldreich, P., Lithwick, Y., \& Sari, R. 2004, ARAA, 42, 549

Gorti, U. \& Hollenbach, D. 2004, ApJ, 613, 424

Gorti, U. \& Hollenbach, D. 2009, ApJ, 690, 1539

Greaves, J. S., Fischer, D. A., \& Wyatt, M. C. 2006, MNRAS, 366, 283

Greaves, J. S., Stauffer, J. R., Collier Cameron, A., Meyer, M. R., \& Sheehan, C. K. W. 2009, MNRAS, 394, L36

Haisch, K. E., Jr., Lada, E. A., \& Lada, C. J. 2001, ApJ, 553, L153

Hartigan, P., Edwards, S., \& Ghandour, L. 1995, ApJ, 452, 736

Hartmann, L. 1998, Accretion processes in star formation / Lee Hartmann. Cambridge, UK; New York : Cambridge University Press, 1998. (Cambridge astrophysics series; 32).

Hartmann, L., Calvet, N., Gullbring, E., \& D'Alessio, P. 1998, ApJ, 495, 385

Hillenbrand, L. A. 2008, Physica Scripta Volume T, 130, 014024

Hillenbrand, L. A., et al. 2008, ApJ, 677, 630

Ida, S. \& Lin, D. N. C. $2008, A p J, 673,487$

Ireland, M. J. \& Kraus, A. L. 2008, ApJ, 678, L59

Jacobsen, S. B. 2005, Geochimica et Cosmochimica Acta Supplement, 69, 386

Johnson, J. A., Butler, R. P., Marcy, G. W., Fischer, D. A., Vogt, S. S., Wright, J. T., \& Peek, K. M. G. 2007, ApJ, 670, 833

Kalas, P., et al. 2008, Science, 322, 1345

Kenyon, S. J. \& Bromley, B. C. 2004, ApJ, 602, L133

Kenyon, S. J. \& Bromley, B. C. 2008, ApJS, 179, 451

Laskar, J. 1994, A\&̈A, 287, L9

Lissauer, J. J. \& Stevenson, D. J. 2007, Protostars and Planets V, 591

Lovis, C., et al. 2006, Nature, 441, 305

Marois, C., Macintosh, B., Barman, T., Zuckerman, B., Song, I., Patience, J., Lafrenière, D., \& Doyon, R. 2008, Science, 322, 1348

Mayor, M., et al. 2009, A\&A, 493, 639

Merín, B., et al. 2008, ApJS, 177, 551

Meyer, M. R., Calvet, N., \& Hillenbrand, L. A. 1997, AJ, 114, 288

Meyer, M. R., et al. 2006, PASP, 118, 1690

Meyer, M. R., Backman, D. E., Weinberger, A. J., \& Wyatt, M. C. 2007, Protostars and Planets $\mathrm{V}, 573$

Meyer, M. R., et al. 2008, ApJ, 673, L181

Monin, J.-L., Clarke, C. J., Prato, L., \& McCabe, C. 2007, Protostars and Planets V, 395

Moro-Martín, A., et al. 2007a, ApJ, 658, 1312 
Moro-Martín, A., et al. 2007b, ApJ, 668, 1165

Murison, M. A., Lecar, M., \& Franklin, F. A. 1994, AJ, 108, 2323

Muzerolle, J., Calvet, N., Hartmann, L., \& D’Alessio, P. 2003, ApJ, 597, L149

Nagasawa, M., Thommes, E. W., Kenyon, S. J., Bromley, B. C., \& Lin, D. N. C. 2007, Protostars and Planets V, 639

Najita, J. R., Strom, S. E., \& Muzerolle, J. 2007, MNRAS, 378, 369

Pascucci, I., et al. 2006, ApJ, 651, 1177

Pascucci, I., Apai, D., Hardegree-Ullman, E. E., Kim, J. S., Meyer, M. R., \& Bouwman, J. 2008a, ApJ, 673, 477

Pascucci, I., Apai, D., Luhman, K., Henning, T., Bouwman, J., Meyer, M., Lahuis, F., \& Natta, A. 2009, ApJ, in press (arXiv:0810.2552)

Pontoppidan, K. M., Blake, G. A., van Dishoeck, E. F., Smette, A., Ireland, M. J., \& Brown, J. 2008, ApJ, 684, 1323

Roccatagliata, V., Henning, T., Wolf, S., Rodmann, J., Corder, S., Carpenter, J. M., Meyer, M., \& Dowell, D. 2009, arXiv:0902.0338

Sanders, I. S. \& Taylor, G. J. 2005, Chondrites and the Protoplanetary Disk, 341, 915

Santos, N. C., Israelian, G., \& Mayor, M. 2004, A\&SA, 415, 1153

Scott, E. R. D. 2007, Annual Review of Earth and Planetary Sciences, 35, 577

Siegler, N., Muzerolle, J., Young, E. T., Rieke, G. H., Mamajek, E. E., Trilling, D. E., Gorlova, N., \& Su, K. Y. L. 2007, ApJ, 654, 580

Shu, F., Najita, J., Ostriker, E., Wilkin, F., Ruden, S., \& Lizano, S. 1994, ApJ, 429, 781

Silverstone, M. D., et al. 2006, ApJ, 639, 1138

Skrutskie, M. F., Dutkevitch, D., Strom, S. E., Edwards, S., Strom, K. M., \& Shure, M. A. 1990, $A J, 99,1187$

Strom, K. M., Strom, S. E., Edwards, S., Cabrit, S., \& Skrutskie, M. F. 1989, AJ, 97, 1451

$\mathrm{Su}, \mathrm{K}$. Y. L., et al. 2006, ApJ, 653, 675

Trilling, D. E., et al. 2007, ApJ, 658, 1289

Trilling, D. E., et al. 2008, ApJ, 674, 1086

Tsiganis, K., Gomes, R., Morbidelli, A., \& Levison, H. F. 2005, Nature, 435, 459

Watson, D. M., et al. 2009, ApJS, 180, 84

Weidenschilling, S. J. 1977, MNRAS, 180, 57

Werner, M., Fazio, G., Rieke, G., Roellig, T. L., \& Watson, D. M. 2006, ARAA, 44, 269

Wyatt, M. C., Smith, R., Greaves, J. S., Beichman, C. A., Bryden, G., \& Lisse, C. M. 2007, ApJ, 658, 569

Wyatt, M. C. 2008, ARAA, 46, 339 


\section{Discussion}

M. LiU: Open clusters and young moving groups represent the best "benchmark" systems for understanding evolution, since they are coeval and formed in the same environment. However, even in these very simple samples, debris disk properties are diverse and not easily explained. Do you find this to be a discouraging result in attempting to develop a comprehensive picture of debris disk evolution?

M. MEYER: Evidence from debris disk studies as well observations of exoplanets suggest that the outcomes of the planet formation process are diverse, as are the paths of subsequent evolution. By combining detailed studies of individual objects (those systems that are resolved in scattered light and/or thermal emission) as well as large surveys conducted with ground- and space- based telescopes (Spitzer, Herschel and JWST) we can hopefully discern the overall climates that are conducive to planet formation, in contrast to our inability to predict the prospects of planet formation for any system (the "weather").

R. JEFFrIEs: Could the low frequency of debris disks be explained by "episodic" dust production? In other words, could debris disks be more common but only episodically produced?

M. MEYER: It is true that we only observe the product of the frequency of the debris disk phenomenon and the distribution of durations. Indeed, if the warm debris disk epoch lasts a short time, the overall frequency could be much higher than observed (Meyer et al 2008). However observational support for this scenario is lacking (Carpenter et al. 2009). Wyatt et al. (2007) explore conditions under which an observed debris disk can be unambiguously identified as transient. 


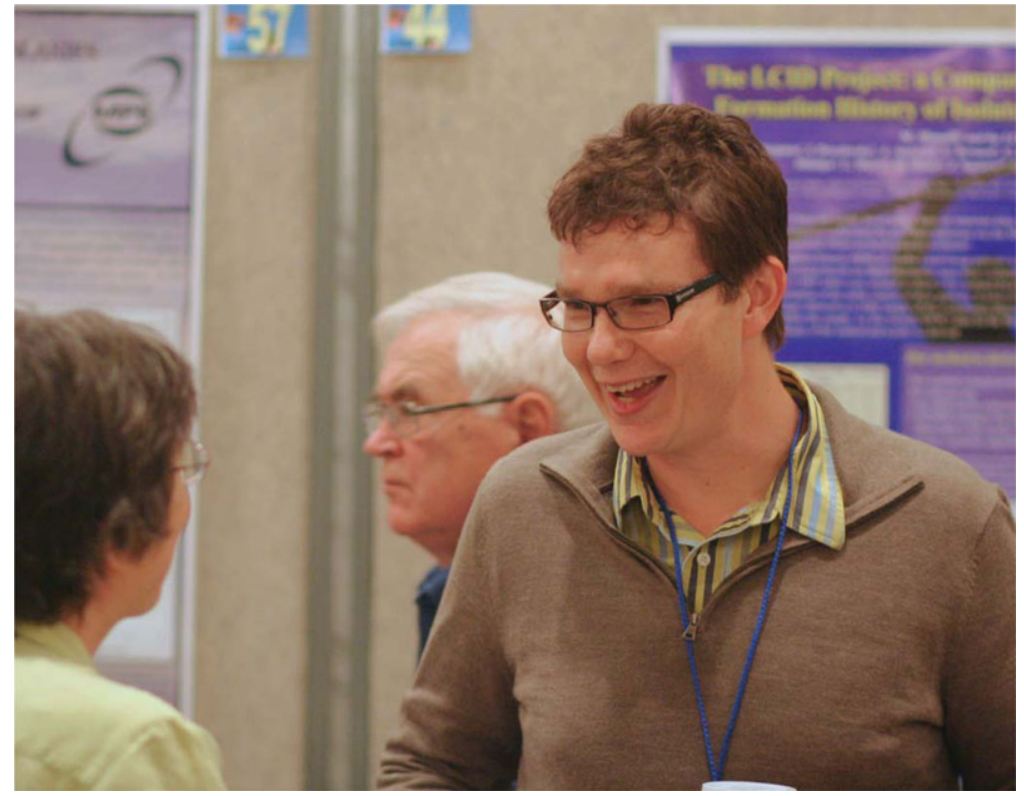

Michael Meyer

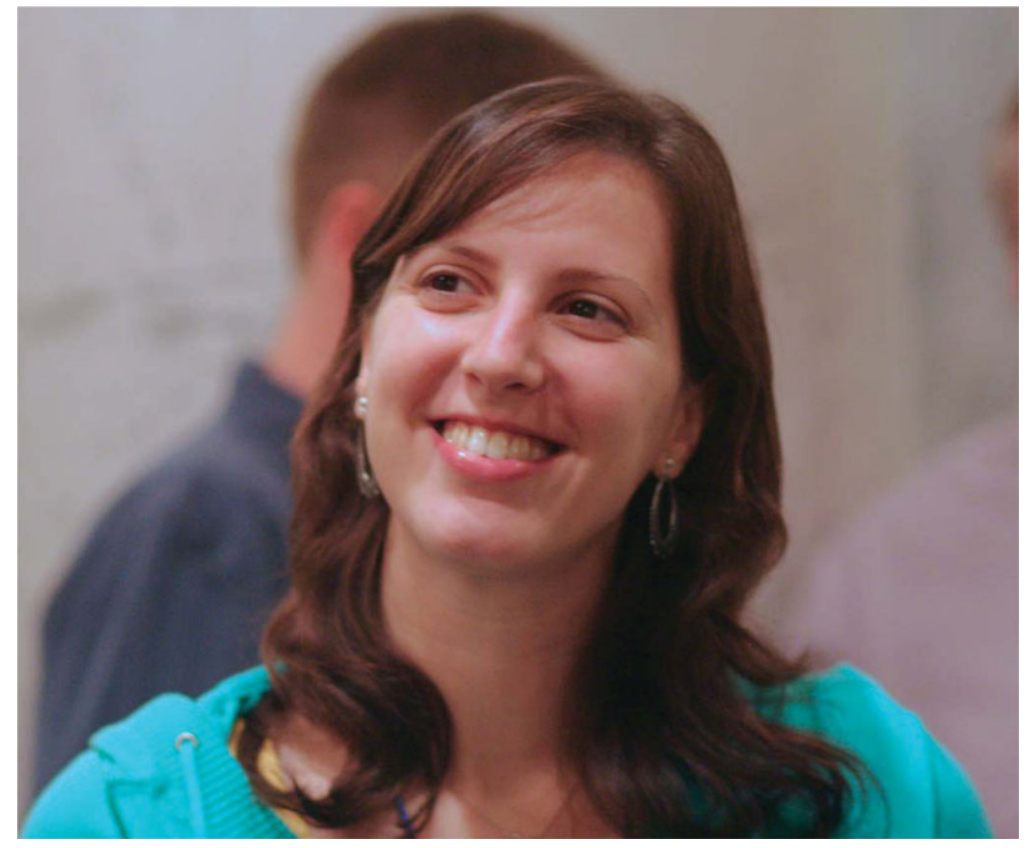

Antonela Monachesi 